\title{
Ácido úrico sérico como marcador de gravedad clínica y comorbilidad en psoriasis en placas
}

\author{
Adameck Abraham Hernández-Collazo, ${ }^{1}$ Guadalupe Villanueva-Quintero, ${ }^{1}$ Marco Antonio Rodríguez- \\ Castellanos, ${ }^{1}$ Eduardo David Poletti-Vázquez ${ }^{2}$ y Anabell Alvarado-Navarro ${ }^{3}$ \\ ${ }^{1}$ Instituto Dermatológico de Jalisco Dr. José Barba Rubio, Jalisco, México; ${ }^{2}$ Universidad Autónoma de Aguascalientes, Aguascalientes; ${ }^{3}$ Centro de \\ Investigación en Inmunología y Dermatología, Jalisco, México
}

\section{Resumen}

Introducción: La hiperuricemia es común en pacientes con psoriasis. Se ha sugerido que la elevación de ácido úrico en psoriasis está fuertemente asociada con morbilidad cardiovascular. Objetivo: Determinar la relación entre niveles de ácido úrico, gravedad clínica medida por Psoriasis Area Severity Index (PASI), Body Surface Area (BSA) y static Physician's Global Assessment (sPGA) en pacientes con psoriasis en placas y comorbilidades ungueales y artritis psoriásica. Método: Determinación de ácido úrico sérico en 45 pacientes con psoriasis en placas y 45 controles pareados por sexo, edad e índice de masa corporal; medición de índices de gravedad clínica en pacientes y presencia de manifestaciones ungueales y articulares. Resultados: Los pacientes con psoriasis presentaron niveles más elevados de ácido úrico (7.03 \pm 1.47 versus $5.32 \pm 1.17$, $p<0.01$ ) y mayor prevalencia de hiperuricemia asintomática (68 versus $17.8 \%, p<0.01$ ) que los controles. Existió correlación proporcional significativa entre gravedad determinada por PASI y ácido úrico $\left(r^{2}=0.70\right)$ y entre manifestaciones articulares e hiperuricemia en pacientes con psoriasis en placas $(p<0.01$; $R M=2.85$, IC $95 \%=1.52-5.33)$. Conclusiones: Los niveles séricos de ácido úrico tuvieron correlación proporcional con PASI y se asociaron con manifestaciones articulares en pacientes con psoriasis en placas.

PALABRAS CLAVE: Hiperuricemia. Psoriasis. Artritis psoriásica. Ácido úrico.

\begin{abstract}
Introduction: Hyperuricemia is common in patients with psoriasis. Uric acid elevation in psoriasis has been suggested to be strongly associated with cardiovascular morbidity. Objective: To determine the relationship between uric acid levels and clinical severity as measured by the Psoriasis Area Severity Index (PASI), body surface area (BSA) and static Physician's Global Assessment ( $S P G A$ ) in patients with plaque psoriasis and nail comorbidities and psoriatic arthritis. Method: Determination of serum uric acid in 45 patients with plaque psoriasis and 45 controls matched by gender, age and body mass index; measurement of patient clinical severity indices and presence of nail and joint manifestations. Results: Patients with psoriasis had higher levels of uric acid ( $7.03 \pm 1.47$ versus $5.32 \pm 1.17, p<0.01)$, and higher prevalence of asymptomatic hyperuricemia than controls $(68 \%$ versus $17.8 \%, p<0.01)$. There was significant proportional correlation between PASI-determined severity and uric acid $\left(r^{2}=0.70\right)$, and between joint manifestations and hyperuricemia in patients with plaque psoriasis $(p<0.01$; $O R=2.85,95 \% \mathrm{Cl}=1.52-5.33)$. Conclusions: Serum uric acid levels had a proportional correlation with PASI and were associated with joint manifestations in patients with plaque psoriasis.
\end{abstract}

KEY WORDS: Hyperuricemia. Psoriasis. Psoriatic arthritis. Uric acid.

Correspondencia:

Adameck Abraham Hernández-Collazo

E-mail: adam_ck11@hotmail.com
Fecha de recepción: 21-06-2017

Fecha de aceptación: 09-11-2017

DOI://dx.doi.org/10.24875/GMM.17003515
Gac Med Mex. 2018;154:427-431

Disponible en PubMed

www.gacetamedicademexico.com 


\section{Introducción}

La psoriasis es una enfermedad crónica autoinflamatoria que afecta a $2 \%$ de la población en países occidentales. ${ }^{1,2}$ Su patogénesis incluye una cascada inmunológica de citocinas proinflamatorias que resultan en un estado de inflamación persistente y recambio celular epidérmico incrementado. ${ }^{3} \mathrm{Su}$ espectro incluye diferentes manifestaciones cutáneas que pueden coexistir en un mismo paciente, si bien todas comparten características esenciales: eritema, engrosamiento y escama. El estado inflamatorio persistente se ha relacionado con manifestaciones extracutáneas: aumento del riesgo cardiovascular, artritis psoriásica secundaria a aumento del estrés oxidativo y decremento de la capacidad antioxidante. El recambio epidérmico está acentuado, con las características clínicas e histopatológicas diagnósticas de la enfermedad. ${ }^{4,5}$ El aumento en el ciclo celular queratinocítico condiciona incremento en el metabolismo purínico, que eleva los niveles de ácido úrico sérico. ${ }^{6}$

Desde 1948, Lobitz y Brunsting describieron por primera vez la relación entre hiperuricemia y psoriasis; ${ }^{7}$ recientemente, Gisondi et al. ${ }^{8}$ confirmaron esta relación, reconociendo a la psoriasis como el factor de riesgo más importante en un análisis multivariable al compararlo con otros factores de riesgo conocidos para hiperuricemia, como obesidad o síndrome metabólico. En 2016, Li et al., en un metaanálisis que evaluó los datos de 14 estudios clínicos e incluyó a 29416 pacientes con psoriasis, concluyeron que la correlación entre psoriasis e hiperuricemia muestra un patrón étnico asociado con componentes genéticos y diferentes estilos de vida. ${ }^{9}$

El aumento del ácido úrico sérico se ha asociado con aumento en el grosor de la íntima carotídea en pacientes con artritis psoriásica; mientras que en pacientes sin psoriasis se ha utilizado como predictor de eventos cardiovasculares y de mortalidad de origen cardiovascular. $^{10}$ Sin embargo, a la fecha no se conoce si la relación entre ácido úrico y eventos cardiovasculares en psoriasis es circunstancial o causal.

La investigación de hiperuricemia como parámetro pronóstico y de valoración clínica en psoriasis es prometedora. Isha et al. demostraron que el tratamiento efectivo de psoriasis durante 12 semanas reduce significativamente los niveles séricos del ácido úrico y la proteína $C$ reactiva, ${ }^{11}$ sin embargo, hasta ahora no existen estudios que evalúen la correlación entre ácido úrico y gravedad clínica en pacientes con psoriasis.

El objetivo de este estudio fue establecer la asociación entre los niveles de ácido úrico con las escalas de gravedad más utilizadas en la práctica clínica, como el Índice de Gravedad y Área en Psoriasis (PASI, Psoriasis Area Severity Index), Superficie de Área Corporal (BSA, Body Surface Area) y Evaluación Estática Global Médica (sPGA, static Physician Global Assessment); además de su asociación con otros parámetros de gravedad clínica como dislipidemia, manifestaciones articulares y ungueales. Asimismo, se compararon los niveles de ácido úrico y prevalencia de hiperuricemia en pacientes con psoriasis y sujetos control.

\section{Métodos}

Se incluyeron 45 pacientes, reclutados de forma consecutiva y que acudieron por primera vez a la clínica de psoriasis del Instituto Dermatológico de Jalisco Dr. José Barba Rubio, entre noviembre de 2014 y enero de 2015. Se incluyeron todos los pacientes con diagnóstico clínico e histopatológico de psoriasis y que no recibieron tratamiento por al menos tres meses antes de la investigación. El grupo control estuvo integrado por 45 personas sanas, seleccionadas para ser pareadas de acuerdo con la edad, el sexo y el índice de masa corporal con los pacientes con psoriasis. Tanto en el grupo control como en el de pacientes no fueron incluidos los individuos con consumo de $>2$ bebidas por semana, evaluado a través de cuestionario estandarizado, dietas hiperproteínicas o catabólicas, enfermedad renal crónica avanzada, medicamentos que alteran el metabolismo del ácido úrico (alopurinol, diuréticos, etambutol, pirazinamida, ketoconazol, salicilatos, teofilina) y antecedente familiar o personal de gota.

Cada paciente fue evaluado por un médico dermatólogo para medir la gravedad clínica mediante PASI, BSA y SPGA, y valorado para el diagnóstico de artritis psoriásica usando los Criterios de Clasificación para Artritis Psoriásica (CASPAR, Classification Criteria for Psoriatic Arthritis). BSA se basa en la medición de la superficie afectada por las lesiones de psoriasis, usando la palma de la mano del paciente (incluyendo los dedos), lo que equivale a $1 \%$ de la totalidad de la superficie corporal. ${ }^{12}$

La determinación de SPGA emplea una escala subjetiva de puntuación de intensidad de las lesiones sin valorar la extensión, con una escala del cero al seis; 
se aplica el valor mínimo a la ausencia de lesiones o lesiones blanqueadas y la puntuación aumenta ante infiltración, eritema y escama, que se identifica con el máximo valor. ${ }^{13}$

El índice PASI se establece con la valoración de cada lesión de psoriasis, al otorgar un valor de cero a cuatro a cada una de las tres características de la lesión elemental de acuerdo con su gravedad: eritema, infiltración y escama. Este resultado se combina con una evaluación ponderada del área afectada de cada segmento del cuerpo: cabeza y cuello, tronco, extremidades superiores e inferiores..$^{12,13}$

En cuanto a los criterios de CASPAR para clasificación de artritis psoriásica, se debe confirmar en el paciente la presencia de artropatía periférica, axial o entesitis y tres puntos de alguna de las manifestaciones clínicas, serológicas o radiológicas de la enfermedad. ${ }^{14}$

Se determinó la presencia de manifestaciones ungueales en pacientes con psoriasis: onicolisis, hoyuelos, leuconiquia, mancha en aceite y mancha en salmón. Se obtuvo muestra de $3.5 \mathrm{~mL}$ de sangre periférica en ayuno de 12 horas tanto de pacientes como de controles para determinación de ácido úrico sérico mediante un espectrofotómetro marca Varian modelo SpectrAA-200. Se definió hiperuricemia en los individuos con rangos de ácido úrico sérico mayores a $6.5 \mathrm{mg} / \mathrm{dL}$ para el sexo masculino y $6.0 \mathrm{mg} / \mathrm{dL}$ para el femenino.

Se utilizaron medidas de tendencia central, estadística descriptiva, $t$ de Student y correlación de Pearson mediante el programa estadístico OpenEpi versión 3.01.

\section{Resultados}

Se obtuvo una muestra de 45 pacientes y 45 controles sanos pareados por edad, sexo e índice de masa corporal. En uno y otro grupo hubo 29 hombres y 16 mujeres; la media de edad fue de $49.38 \pm 17.36$ años en el grupo de pacientes y $50.98 \pm 17.3$ años en el grupo control; el índice de masa corporal en los pacientes tuvo una media de $31.18 \pm 6.66 \mathrm{~kg} / \mathrm{m}^{2}$ y en el grupo control de $30.15 \pm 6.08 \mathrm{~kg} / \mathrm{m}^{2}$. Las características epidemiológicas se describen en la Tabla 1. En los pacientes con psoriasis, la media estadística para PASI fue de $10.90 \pm 5.82$, para BSA de $28.26 \pm 15.26 \%$ y para sPGA de $2.96 \pm 2.38$. De los 45 pacientes, seis no acudieron a consulta para diagnóstico de artritis psoriásica o acudieron sin radiografía de manos o pies o factor reumatoide sérico; 39 fueron evaluados para su clasificación, de los cuales
Tabla 1. Características demográficas, somatométricas y bioquímicas en individuos con y sin psoriasis

\begin{tabular}{lcc}
\hline Grupo & $\begin{array}{c}\text { Psoriasis } \\
(\mathbf{n}=\mathbf{4 5})\end{array}$ & $\begin{array}{c}\text { Control } \\
(\mathbf{n}=\mathbf{4 5})\end{array}$ \\
\hline Relación hombres/mujeres $(\mathrm{n})$ & $29 / 16$ & $29 / 16$ \\
Frecuencia de hiperuricemia $(\mathrm{n})$ & $31(68.88 \%)$ & $8(17.88 \%)$ \\
\hline Edad (años) & Media $\pm \mathbf{D E}$ & Media $\pm \mathbf{D E}$ \\
\hline Índice de masa corporal $\left(\mathrm{kg} / \mathrm{m}^{2}\right)$ & $31.18 \pm 6.66$ & $30.15 \pm 6.08$ \\
Ácido úrico sérico $(\mathrm{mg} / \mathrm{dL})$ & $7.03 \pm 1.47$ & $5.32 \pm 1.17$ \\
\hline
\end{tabular}

$53 \%(21 / 39)$ presentaron manifestaciones articulares que cumplieron con los criterios de CASPAR.

Significativamente los pacientes con psoriasis tuvieron niveles mayores de ácido úrico que los sujetos del grupo control $(7.03 \pm 1.47 \mathrm{mg} / \mathrm{dL}$ versus $5.32, \pm 1.17 \mathrm{mg} / \mathrm{dL}, \mathrm{p}<0.01)$. La frecuencia de hiperuricemia fue mayor en el grupo de psoriasis que en el control (68.88 versus $17.8 \%, p<0.01$ ), con una diferencia significativa. Estos resultados se mantuvieron al estratificar a los pacientes por sexo; en este caso, tanto hombres como mujeres con psoriasis tuvieron ácido úrico mayor que su contraparte control. De igual manera, ante la presencia de síndrome metabólico, los pacientes con psoriasis tuvieron mayor nivel de ácido úrico que el grupo control.

En la Figura 1 se aprecia que con el coeficiente de correlación de Pearson se encontró correlación fuertemente positiva entre medición de PASI y niveles de ácido úrico sérico $\left(r^{2}=70, p<0.01\right)$. En contraparte, la medición de gravedad medida por BSA $\left(r^{2}=0.32\right)$ y sPGA $\left(r^{2}=0.29\right)$ tuvo una correlación débilmente positiva (Figuras 2 y 3 ). En cuanto a otros índices de gravedad, $57.77 \%(26 / 45)$ de los pacientes tuvo manifestaciones ungueales, principalmente onicolisis; de ellos, 69 \% (18/26) presentó hiperuricemia. Con las tablas de contingencia y prueba de chi cuadrado no se identificó asociación significativa $(p=0.43)$.

En la Tabla 2 se detallan las variables estudiadas en el grupo de pacientes con psoriasis que presentaron hiperuricemia (31/45) versus aquellos con niveles de ácido úrico normal respecto a su sexo y psoriasis (14/45). En cuanto a la edad, sexo, índice de masa corporal, así como niveles de colesterol total o triglicéridos, no se encontraron diferencias estadísticas entre el grupo con psoriasis e hiperuricemia versus pacientes con psoriasis y ácido úrico normal. La gravedad evaluada por PASI fue mayor en pacientes con hiperuricemia comparada con la de aquellos con 


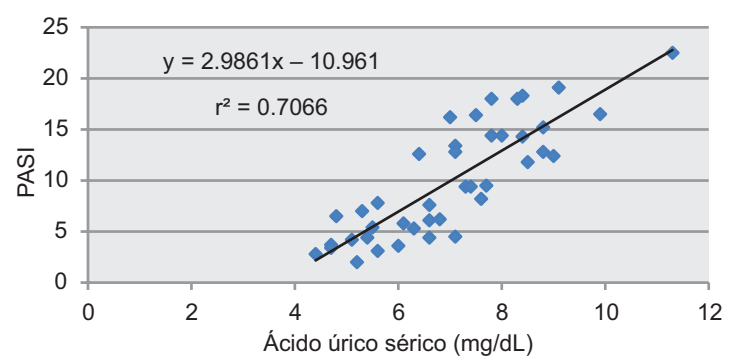

Figura 1. Correlación de Pearson. $P A S I$ y ácido úrico sérico $\left(r^{2}=0.70\right.$, $p<0.01) . P A S I=$ Psoriasis Area and Severity Index

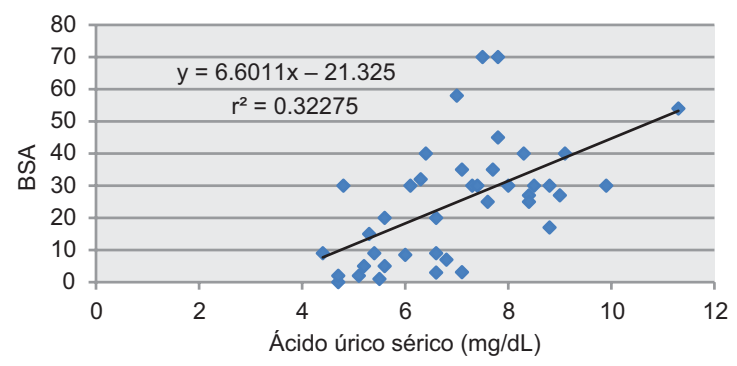

Figura 2. Correlación de Pearson: $B S A$ y ácido úrico sérico $\left(r^{2}=0.32\right.$, $p<0.01) . B S A=$ Body Surface Area.

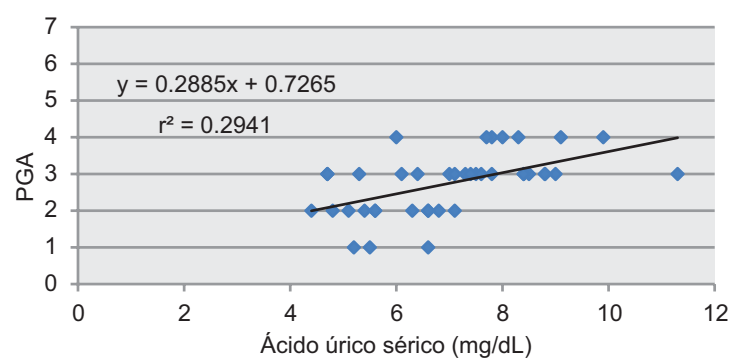

Figura 3. Correlación de Pearson: $S P G A$ y ácido úrico sérico $\left(r^{2}=0.29\right.$, $p$ < 0.01). sPGA = Static Physician's Global Assessment.

psoriasis sin hiperuricemia (10.90 \pm 5.81 versus $5.41 \pm 2.76)$, con diferencia significativa $(p=0.007)$. La gravedad medida por BSA fue mayor en pacientes con hiperuricemia comparada con la de aquellos con psoriasis sin hiperuricemia $(28.26 \pm 19.05$ versus $14.18 \pm 12.49$ ), sin embargo, esta diferencia no fue estadísticamente significativa entre ambos grupos $(p=0.16)$. Asimismo, la gravedad medida por sPGA no demostró diferencia significativa entre ambos grupos $(2.97 \pm 0.76$ versus $2.21 \pm 0.80, p=0.95)$.

Finalmente, $81 \%$ de los pacientes con manifestaciones articulares que cumplieron los criterios de CASPAR presentaron hiperuricemia (17/21). Mediante el mismo análisis estadístico se identificó asociación estadísticamente significativa entre la presencia de artritis psoriásica e hiperuricemia $(\mathrm{p}<0.01, \mathrm{RM}=2.85$, IC $95 \%=1.52-5.33)$.

\section{Discusión}

La frecuencia de hiperuricemia asintomática fue cuatro veces mayor en pacientes con psoriasis en placas que en el grupo control (69 versus $17 \%$ ), superior a lo reportado por Gisondi en Italia en 2014, quien describió que fue solo tres veces mayor que en el grupo control (19 versus $7 \%$ ). ${ }^{8}$ Resalta que nuestra población control presentó frecuencia de hiperuricemia tan alta como la de los pacientes con psoriasis del estudio de Gisondi. ${ }^{8}$ Sin embargo, nuestro grupo control tuvo una frecuencia de hiperuricemia similar a la esperado en población mexicana sin psoriasis (Llamazares, $19 \%$ ). ${ }^{15}$ Esto concuerda con lo descrito por Li en un metaanálisis de 2016, en el cual la asociación con hiperuricemia se relacionó con un componente étnico. ${ }^{7}$

En el grupo de pacientes con psoriasis no hubo diferencia estadísticamente significativa respecto al sexo, edad, índice de masa corporal, dislipidemia y alteraciones ungueales entre el subgrupo con hiperuricemia comparado con el que tuvo niveles normales de ácido úrico. Este es el primer estudio realizado sobre ácido úrico en población mexicana con psoriasis en placas.

Conforme a lo esperado, los pacientes que presentaron niveles de hiperuricemia de acuerdo con su sexo tuvieron gravedad más alta medida por PASI. Lo anterior se relaciona aún más al utilizar el coeficiente de correlación logística de Pearson. Por otro lado, la poca correlación entre ácido úrico y BSA como escala de gravedad clínica, comparada con la correlación proporcional encontrada con PASI sugiere que la fisiopatogenia de la hiperuricemia no solo depende de una afección extensa, sino también de características específicas de inflamación de la enfermedad, asimismo, que el ácido úrico puede servir como una escala objetiva para la valoración de la gravedad y la respuesta al tratamiento.

Acerca de la artritis psoriásica, lo reportado indica que el ácido úrico es un marcador sérico de daño articular en pacientes con psoriasis en placas y subraya la necesidad de la medición de ácido úrico de forma rutinaria. Por lo tanto, los resultados de este estudio indican que la hiperuricemia incrementa el riesgo de artritis psoriásica casi tres veces.

Aun cuando no se encontró relación entre ácido úrico sérico y riesgo cardiovascular en psoriasis, esta se ha demostrado en otras poblaciones, por lo que se sugieren otros estudios con muestras de mayor tamaño. Asimismo, se necesita ahondar en los mecanismos 
Tabla 2. Características estadísticas y variables estudiadas en pacientes con psoriasis en placas

\begin{tabular}{|c|c|c|c|c|c|}
\hline \multirow{3}{*}{$\begin{array}{l}\text { Variable } \\
\text { Sexo (masculino/femenino) }\end{array}$} & \multicolumn{2}{|c|}{ Ácido úrico normal $(n=14)$} & \multicolumn{2}{|c|}{ Hiperuricemia $(n=31)$} & \multirow{3}{*}{$\frac{\mathbf{p}}{0.08}$} \\
\hline & \multicolumn{2}{|c|}{$7 / 7$} & \multicolumn{2}{|c|}{$22 / 9$} & \\
\hline & $\mathbf{n}$ & $\%$ & $\mathrm{n}$ & $\%$ & \\
\hline Hipertensión arterial, n (\%) & 2 & 14.2 & 8 & 35.4 & 0.07 \\
\hline Alteraciones ungueales, $n(\%)$ & 8 & 57.1 & 18 & 58 & 0.43 \\
\hline Artritis psoriásica, n (\%) & 4 & 28.5 & 17 & 54.8 & 0.01 \\
\hline
\end{tabular}

\begin{tabular}{|c|c|c|c|}
\hline & Media $\pm \mathrm{DE}$ & Media $\pm \mathrm{DE}$ & \\
\hline Edad, años & $52.69 \pm 17.62$ & $49.58 \pm 17.54$ & 0.93 \\
\hline Índice de masa corporal $\left(\mathrm{kg} / \mathrm{m}^{2}\right)$ & $32.42 \pm 7.32$ & $32.54 \pm 7.66$ & 0.90 \\
\hline PASI & $5.82 \pm 2.80$ & $10.90 \pm 5.81$ & 0.007 \\
\hline BSA (\%) & $15.26 \pm 13.26$ & $28.26 \pm 19.06$ & 0.16 \\
\hline PGA & $2.38 \pm 0.76$ & $2.96 \pm 0.76$ & 0.95 \\
\hline Colesterol total (mg/dL) & $196.23 \pm 65.98$ & $193.08 \pm 40.39$ & 0.02 \\
\hline Triglicéridos (mg/dL) & $165.38 \pm 105.28$ & $203.28 \pm 96.6$ & 0.66 \\
\hline
\end{tabular}

Las diferencias se estudiaron mediante t de Student y prueba de chi cuadrado (variables categóricas). PASI = Psoriasis Area and Severity Index, BSA = Body Surface Area,

PGA = Physician Global Assessment

específicos del desarrollo de hiperuricemia en psoriasis y en su utilidad como predictor de enfermedad cardiovascular y renal en pacientes con psoriasis, por modesta que sea su expresión clínica.

\section{Referencias}

1. Gudjonsson JE, Elder JT. Psoriasis: Epidemiology. Clin Dermatol. 2007;25(6):535-546.

2. Takeshita J, Grewal S, Langan SM, Mehta NN, Ogdie A, Van-Voorhees AS, et al. Psoriasis and comorbid diseases: Epidemiology. J Am Acad Dermatol. 2017;76(3):377-390.

3. Krueger J, Bowcock A. Psoriasis pathophysiology: Current concepts of pathogenesis. Ann Rheum Dis. 2005;64(Suppl 2):ii30-ii36.

4. Rivas-Bejarano JJ, Valdecantos WC. Psoriasis as autoinflammatory disease. Dermatol Clin. 2013;31(3):445-460.

5. Takeshita J, Grewal S, Langan SM, Mehta NN, Ogdie A, Van-Voorhees AS, et al. Psoriasis and comorbid diseases: implications for management. J Am Acad Dermatol. 2017;76(3):393-403.

6. Kwon HH, Kwon IH, Choi JW, Youn JI. Cross-sectional study on the correlation of serum uric acid with disease severity in Korean patients with psoriasis. Clin Exp Dermatol. 2011;36:473-478.
7. Lobitz W, Brunsting L. Unpublished studies. En: Ormsby OS, Montgomery H. Diseases of the skin. USA: Lea and Febiger; 1948.

8. Gisondi P, Targher G, Cagalli A, Girolomi G. Hyperuricemia in patients with chronic plaque psoriasis. J Am Acad Dermatol. 2014;70(1):127-130.

9. Li X, Miao X, Wang H, Wang Y, Li F, Yang Q, et al. Association of serum uric acid levels in psoriasis: a systematic review and meta-analysis. Medicine (Baltimore). 2016;95(19):e3676.

10. González-Gay MA, González-Juanatey C, Vázquez-Rodríguez TR, Gómez-Acebo I, Miranda-Filloy JA, Paz-Carreira J, et al. Asymptomatic hyperuricemia and serum uric acid concentration correlate with subclinical atherosclerosis in psoriatic arthritis patients without clinically evident cardiovascular disease. Semin Arthritis Rheum. 2009;39(3):157-162.

11. Isha, Jain VK, Lal H. C-reactive protein and uric acid levels in patients with psoriasis. Indian J Clin Biochem. 2011;26(3):309-311.

12. Henseler T, Schmitt-Rau K. A comparison between BSA, PASI, PLASI and SAPASI as measures of disease severity and improvement by therapy in patients with psoriasis. Int J Dermatol. 2008;47(10):1019-1023.

13. Langley RG, Ellis CN. Evaluating psoriasis with Psoriasis Area and Severity Index, Psoriasis Global Assessment, and Lattice System Physician's Global Assessment. J Am Acad Dermatol. 2004;51(4):563-569.

14. Torre-Alonso JC. Utilidad y aplicación en la práctica clínica de los criterios de CASPAR. Reumatol Clin. 2010;6:18-21.

15. Llamazares-Azuara L, Rodríguez-Martínez M, De-La-Cruz-Mendoza E, Torres-Ruvalcaba A, Flores-Sánchez J. Prevalencia de hiperuricemia, resistencia a la insulina, y dislipidemia en jóvenes de 17 a 23 años. Bioquimia. 2007;32:134 